\title{
Identidade de modelos hipsométricos para clones de eucalipto na região oriental do Paraguai
}

\author{
Identity of hypsometric models for eucalyptus clones in the eastern region \\ of Paraguay
}

\author{
Hugo Armando Barúa Acosta ${ }^{1}$ (D), Alexandre Techy de Almeida Garrett ${ }^{1}$ (1), \\ Luciano Rodrigo Lanssanova ${ }^{1}$ (D), Andrea Nogueira Dias ${ }^{1}$ (D), Evandro Vagner Tambarussi ${ }^{1}$ (D), \\ Afonso Figueiredo Filho ${ }^{1}$ (D), Fabiane Aparecida Retslaff Guimarães ${ }^{1}$ (D), Oscar Manuel Vera Cabral ${ }^{2}$ \\ ${ }^{1}$ Universidade Estadual do Centro-Oeste - UNICENTRO, Irati, PR, Brasil \\ ${ }^{2}$ Departamento Central, Universidade Nacional de Assunção - UNA, Ciudad de San Lorenzo, Paraguai
}

Como citar: Acosta, H. A. B., Garrett, A. T. A., Lanssanova, L. R., Dias, A. N., Tambarussi, E. V., Figueiredo Filho, A., Guimarães, F. A. R., \& Cabral, O. M. V. (2020). Identidade de modelos hipsométricos para clones de eucalipto na região oriental do Paraguai. Scientia Forestalis, 48(125), e3206. https://doi.org/10.18671/scifor.v48n125.16

\begin{abstract}
Resumo
O desenvolvimento do setor florestal é recente no Paraguai e é baseado em plantios com híbridos de Eucalyptus spp. Dessa maneira, não há literatura sobre o ajuste de modelos de relação hipsométrica, fundamentais para o cálculo do volume. Assim, o presente trabalho teve como objetivo avaliar modelos de relação hipsométrica para diferentes genótipos de eucalipto na região Oriental do Paraguai, e verificar a possibilidade do uso de uma única equação para a relação hipsométrica, por meio de teste de identidade. Os plantios foram avaliados nos distritos de Hernandarias (Local 1) e San Juan Nepomuceno (Local 2), avaliando-se 13 clones no Local 1 e 10 clones no Local 2. Nos plantios foram instaladas parcelas permanentes de $1.000 \mathrm{~m}^{2}$ para avaliar os plantios dos 12 aos 93 meses de idade, medindo todos os diâmetros à altura do peito (DAP) e as alturas totais das árvores, com remedições anuais. Para os dados coletados foram ajustados os modelos de Curtis, Prodan (modificado), Nogueira e Campos. Os ajustes apresentaram coeficiente de determinação ajustado $\left(R^{2}\right.$ adj $)$ variando entre 0,96 e 0,98 , e erro padrão de estimativa em porcentagem ( $\mathrm{S}_{\mathrm{yx}} \%$ ) variando de 5,4 a $11,2 \%$. No Local 1 , os modelos de Nogueira e de Campos apresentaram os melhores ajustes, enquanto no Local 2 o modelo de Campos apresentou o melhor ajuste. Nos dois locais o modelo de Campos apresentou a melhor distribuição gráfica de resíduos, por isso o modelo foi selecionado para o teste de identidade. O teste de identidade apontou que não é possível o uso de uma única equação entre genótipos em cada local, e entre os locais. Concluiu-se que o modelo de Campos, com a variáveis independentes idade, $\mathrm{h}_{\text {dom }}$ e DAP, deve ser ajustado por genótipos e para cada local, tendo uma boa performance para as estimativas de altura de diferentes genótipos de eucalipto.
\end{abstract}

Palavras-chave: Curvas h/d; Genótipos de Eucalyptus spp.; Modelagem; Teste de identidade.

\begin{abstract}
In Paraguay, the development of the forestry sector is relatively recent and is based on hybrid Eucalyptus spp. plantations. As such, no information about the adjustment of hypsometric relationship models is available, which is essential for volume calculation. Thus, the present study aims to evaluate hypsometric relationship models for different eucalypt genotypes in Eastern Paraguay and determine the possibility of a single equation for the hypsometric relationship through an identity test. The evaluated plantation areas were located in Hernandarias (Location 1) and San Juan Nepomuceno (Location 2), with 13 clones evaluated in Location 1 and 10 clones in Location 2. In the plantation areas, permanent inventory plots of $1,000 \mathrm{~m}^{2}$ were measured to evaluate the plantations from 12- to 93-months after planting, including annual remeasurement of diameter at breast height $(\mathrm{DBH})$ and total tree height. For the collected data,
\end{abstract}

Fonte de financiamento: Coordenadoria de Aperfeiçoamento de Pessoal de Nível Superior (CAPES).

Conflito de interesse: Nada a declarar.

Autor correspondente: armandobarua@gmail.com

Recebido: 5 dezembro 2018.

Aceito: 7 junho 2019.

Editor: Francides Gomes da Silva Júnior.

(c) (i) Este é um artigo publicado em acesso aberto (Open Access) sob a licença Creative Commons Attribution, que permite uso, distribuição e reprodução em qualquer meio, sem restrições desde que o trabalho original seja corretamente citado. 
models developed by Curtis, Prodan (modified), Nogueira, and Campos were adjusted. The results showed adjusted coefficient of determination $\left(R^{2}\right.$ adj) varying from 0.96 to 0.98 , and standard error of estimate in percentage $\left(\mathrm{S}_{\mathrm{yx}} \%\right)$ ranging from 5.4 and $11.2 \%$. In Location 1, the Nogueira and Campos models presented the best adjustment, whereas in Location 2 the Campos model presented better results. For both locations, the Campos model showed better residual distribution and was therefore selected for the identity test. The identity test indicated that the use of a single equation among genotypes in each location and between locations was not possible. Therefore, the Campos model, with the independent variables of age, dominant height, and $\mathrm{DBH}$, must be adjusted for genotype and location, but offers good performance to estimate the total height of different genotypes of eucalypts.

Keywords: H/d curves; Eucalyptus spp. genotypes; Modelling; Identity test.

\section{INTRODUÇÃO}

Até recentemente, o setor de florestas do Paraguai era baseado na exploração de florestas nativas (Frey, 2007). Os plantios florestais no país iniciaram-se somente a partir deste século e, em decorrência disto, há uma carência de informações acerca da área efetivamente plantada, utilização dos produtos advindos desses plantios e da produtividade das florestas. Espera-se, todavia, que o retorno econômico de plantios de eucaliptos no país seja similar aos observados no Brasil e Uruguai (Frey, 2007; Portillo, 2010).

No Paraguai, $85 \%$ da área de plantios florestais é representada pelo gênero Eucalyptus spp., havendo também plantios das espécies Toona ciliata M. e Melia spp., (Food and Agriculture Organization of the United Nations, 2002; Portillo, 2010; Britos \& Leguizamón, 2013). Existem também plantios de Pinus spp., mas sem informações disponíveis sobre a área plantada.

O aumento das áreas de florestas plantadas foi expressivo no período de 2010 a 2015, com um aumento de 56.000 ha para 98.000 ha (Food and Agriculture Organization of the United Nations, 2018). O Paraguai possui clima favorável aos plantios de Eucalyptus spp., e a recente expansão das áreas de plantios podem representar aumento de emprego e renda (Instituto Forestal Nacional, 2013). No início da implantação de florestas de eucaliptos era esperado um incremento de 20 a $35 \mathrm{~m}^{3}$. ha $a^{-1}$.ano-1 (Food and Agriculture Organization of the United Nations, 2002), mas atualmente não há disponibilidade na literatura sobre dados de crescimento desses plantios.

Em povoamentos florestais o monitoramento do crescimento e da produção é crucial para o manejo florestal (Ribeiro et al., 2010). Para tal, as principais variáveis necessárias para monitorar a produção são o diâmetro, altura, área basal e volume, que são em geral definidos por amostragem no inventário florestal. Neste contexto, é necessário se conhecer o diâmetro e a altura das árvores para a estimativa de volume (Campos \& Leite, 2009; Sanquetta et al., 2009; Moraes Neto et al., 2010; Sousa et al., 2013), além disso a altura é importante para determinar os índices de sítio (Machado \& Figueiredo Filho, 2006).

No inventário florestal todos os diâmetros das árvores das parcelas são medidos. Por outro lado, a altura, é avaliada apenas para algumas árvores das parcelas, principalmente pelos custos e tempo com esta operação (Sanquetta et al., 2009; Ribeiro et al., 2010; Moraes Neto et al., 2010; Jesus et al., 2015; Scolforo et al., 2015). A partir dos pares de dados de altura e diâmetro são estabelecidas relações hipsométricas, que explicam a relação altura e diâmetro, para determinar a altura das demais árvores (Machado \& Figueiredo Filho, 2006; Sanquetta et al., 2009; Ribeiro et al., 2010; Scolforo et al., 2015).

Diversos fatores influenciam o ajuste de modelos de relação hipsométrica como posição sociológica, região, idade, densidade de plantio e silvicultura (Ribeiro et al., 2010). Apesar de a altura poder ser estimada somente com base nos dados de diâmetro à altura do peito (DAP) como variável independente, dados de altura dominante, sítio e idade podem ser incluídos nos modelos (Leite \& Andrade, 2003; Campos \& Leite, 2009; Sanquetta et al., 2009; Moraes Neto et al., 2010).

Devido a disponibilidade de diferentes modelos hipsométricos, que estão associados à redução de custos, ganhos de acuracidade de estimativas e agilidade de coleta de dados, a avaliação do uso de um mesmo modelo adequado para dados oriundos de diferentes povoamentos é importante para facilitar o planejamento e o manejo florestal. Para essa finalidade, o teste de identidade visa identificar se uma mesma equação pode ser aplicada à 
diferentes grupos de dados, como aplicado para modelos hipsométricos e volumétricos (Moraes Neto et al., 2010; Bonete et al., 2016; Rodrigues et al., 2017).

Assim, devido à falta de informações na literatura sobre o ajuste de modelos hipsométricos no Paraguai, o presente estudo avaliou quatro modelos hipsométricos para diferentes grupos de genótipos de eucalipto em dois locais da região Oriental do Paraguai, e ainda a possibilidade de uso de uma única equação para a relação hipsométrica entre os genótipos e os locais avaliados.

\section{MATERIAL E MÉTODOS}

\section{Caracterização do local}

O presente estudo foi realizado com dados de povoamentos clonais de Eucalyptus spp. da empresa Desarrollos Madereros S.A., localizados na região Oriental do Paraguai, nos distritos de Hernandarias, Departamento de Alto Paraná (Local 1), e no distrito de San Juan Nepomuceno, Departamento de Caazapá (Local 2), como ilustrado na Figura 1. De acordo com a classificação climática de Köppen o clima da região é Cfa (subtropical úmido), com temperatura média de $21,4^{\circ} \mathrm{C}$ em ambos locais, e com precipitação média anual de $1.695 \mathrm{~mm}$ (Local 1) e $1.549 \mathrm{~mm}$ (Local 2).

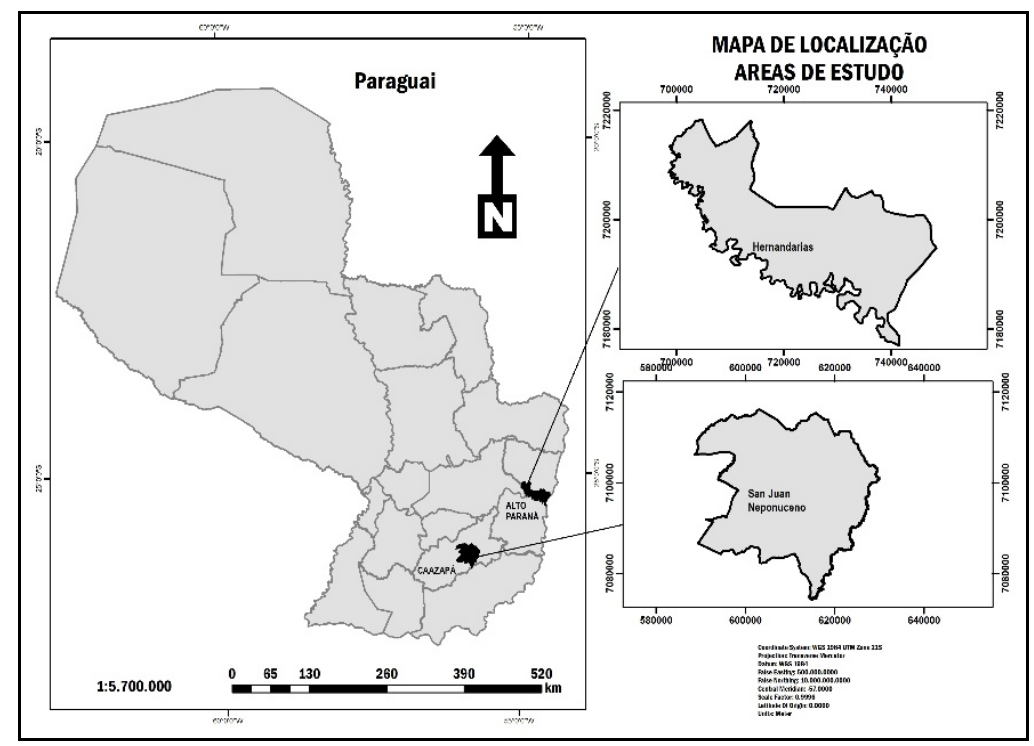

Figura 1. Localização das áreas de estudo na região Oriental do Paraguai.

O manejo aplicado para os plantios clonais de Eucalyptus spp. é o mesmo nos dois locais, e é caracterizado por uma baixa densidade inicial de 620 árvores por hectare, em um espaçamento de 6,45 m entre linhas e 2,5 m entre plantas. Nestas áreas foram aplicados dois desbastes seletivos, o primeiro aos 30 meses, de 33,3\%, e o segundo aos 63 meses de $44 \%$, restando para o corte final, em média, 230 árvores por hectare entre os 84 e 96 meses. Nos plantios foram realizadas cinco podas, sendo a primeira poda aplicada aos 10 meses, com o intuito de se obter na segunda poda uma altura livre de nós de 2,5 m em todas as árvores; a terceira poda ocorreu somente nas árvores selecionadas para o corte final, atingindo 4,3 m livres de nós. A quarta poda proporcionou madeira livre de nós até 6,3 $\mathrm{m}$ de altura, e a última poda (quinta) atingiu 8,3 $\mathrm{m}$ de altura de madeira livre de nós (Figura 2 ) que, dependendo do clone, foi aplicado entre 30 e 36 meses. O manejo dos plantios visou fornecer madeira de qualidade elevada para as indústrias de laminação, serraria e postes tratados. 


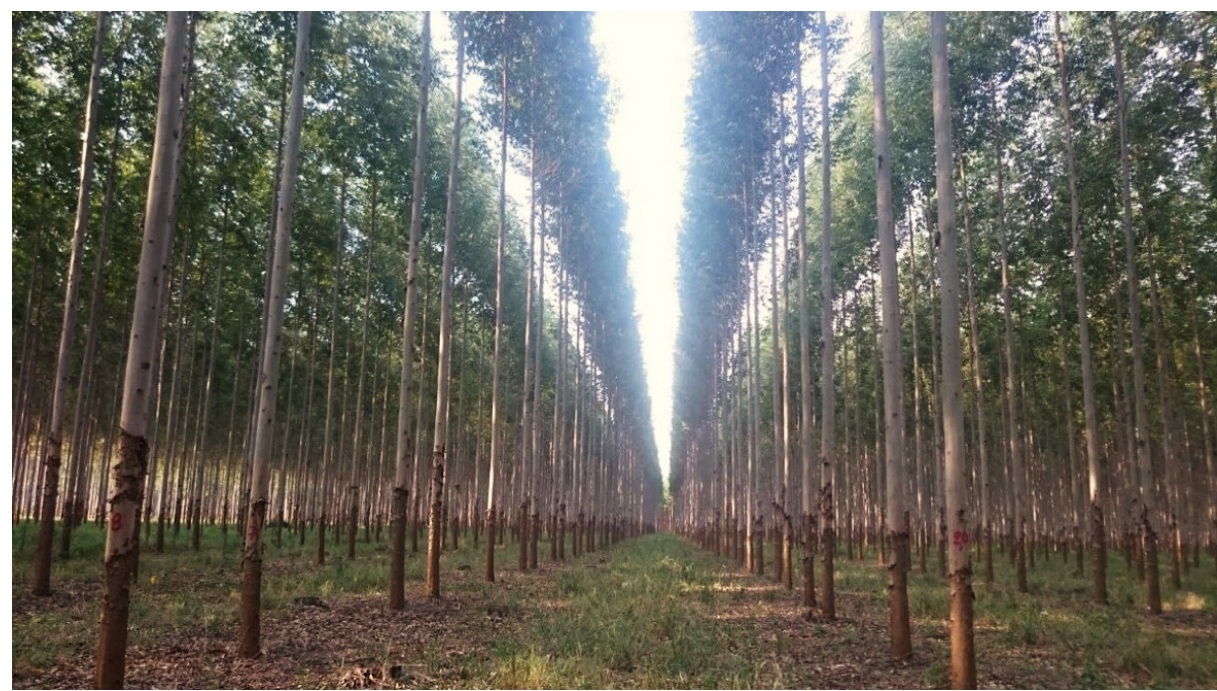

Figura 2. Área de plantio de Eucalyptus spp. avaliada neste estudo, com manejo de desbaste e poda aplicado em Hernardarias (Local 1), Paraguai.

\section{Obtenção dos dados}

Os dados utilizados são oriundos de parcelas permanentes instaladas em formato circular, com $1.000 \mathrm{~m}^{2}$ de área, sendo 36 parcelas permanentes no Local 1 e 43 parcelas no Local 2. A partir da instalação das parcelas aos 12 meses de idade foram coletados os diâmetros à altura do peito (DAP), a 1,30 m do solo, e a altura total (h) de todas as árvores das parcelas com o hipsômetro Vertex. A partir dos dados coletados, a altura dominante $\left(\mathrm{h}_{\mathrm{dom}}\right)$ foi determinada de acordo com a metodologia de Assmann, como indicado por Finger (1992). A remedição das parcelas foi realizada anualmente até o corte final dos plantios.

Foram avaliados 13 clones no Local 1 e 10 clones no Local 2. No Local 1 foram avaliados cinco clones de Eucalyptus grandis, quatro clones do híbrido Eucalyptus grandis x Eucalyptus urophylla e quatro clones do híbrido Eucalyptus urophylla $\times$ Eucalyptus grandis. No local 2 os dados foram obtidos de cinco clones de Eucalyptus grandis, três clones do híbrido Eucalyptus grandis $\mathrm{x}$ Eucalyptus urophylla e dois clones do híbrido Eucalyptus urophylla $\mathrm{x}$ Eucalyptus grandis. A idade das avaliações variou entre locais e clones. No Local 1 os clones de E. grandis e E. grandis $\times$ E. urophylla foram avaliados de 12 a 75 meses, enquanto os clones de $E$. urophylla $x$ E. grandis foram avaliados de 12 a 87 meses. No Local 2 os clones de E. grandis foram avaliados de 12 a 90 meses, para os demais clones as avaliações foram de 12 a 93 meses. Os clones foram agrupados de acordo com cada progênie, formando 3 grupos para cada local: Grupo 1: E. grandis; Grupo 2: E. grandis x E. urophylla; Grupo 3: E. urophylla x E. grandis, como apresentado na Tabela 1.

Tabela 1. Número de clones, diferentes idades e número de observações de pares altura total e diâmetro à altura do peito (h/d) para cada grupo de clones de cada genótipo no Local 1 e Local 2 no Paraguai.

\begin{tabular}{|c|c|c|c|c|c|c|}
\hline Locais & Local & Hernanda & & Local 2 & S. J. Nepon & Iceno) \\
\hline Grupos de Genótipos & $\begin{array}{c}\text { Número de } \\
\text { Clones }\end{array}$ & $\begin{array}{l}\text { Idades } \\
\text { (meses) }\end{array}$ & $\begin{array}{c}\text { Pares } \\
\text { h/d }\end{array}$ & $\begin{array}{c}\text { Número de } \\
\text { Clones }\end{array}$ & $\begin{array}{c}\text { Idades } \\
\text { (meses) }\end{array}$ & Pares h/d \\
\hline $\begin{array}{l}\text { E. grandis } \\
\text { Grupo } 1\end{array}$ & 5 & 12 até 75 & 2333 & 5 & 12 até 90 & 1959 \\
\hline $\begin{array}{l}\text { E. urophylla x E. } \\
\text { grandis } \\
\text { Grupo } 2\end{array}$ & 4 & 12 até 75 & 1585 & 3 & 12 até 93 & 2135 \\
\hline $\begin{array}{c}\text { E. grandis x E. } \\
\text { urophylla } \\
\text { Grupo } 3\end{array}$ & 4 & 12 até 87 & 2154 & 2 & 12 até 93 & 3047 \\
\hline
\end{tabular}




\section{Ajuste dos modelos hipsométricos}

Foram ajustados quatro modelos hipsométricos (Curtis (1967), equação 1, Prodan modificado (Santana (2008), equação 2, Nogueira (2003), equação 3, e Campos et al. (1984)), equação 4, apresentados na Tabela 2), separadamente, para cada grupo (3) de genótipo e em cada local avaliado, totalizando 24 ajustes. Para o ajuste dos modelos, utilizaram-se os dados de DAP, $h, l, h_{\text {dom }}$ provenientes do banco de dados das parcelas permanentes.

Tabela 2. Modelos de relação hipsométrica ajustados para clones de Eucalyptus spp. na região oriental do Paraguai.

\begin{tabular}{ccc}
\hline Autor & Modelo & $\mathbf{N}^{\mathbf{0}}$ \\
\hline Curtis (1967) & $\ln (\mathrm{h})=\beta_{0}+\beta_{1} \mathrm{DAP}^{-1}+\beta_{2} \mathrm{I}^{-1}+\beta_{3}\left(\mathrm{DAP}^{-1} \mathrm{I}\right)+\varepsilon_{\mathrm{i}}$ \\
$\begin{array}{c}\text { Prodan modificado } \\
\text { (Santana, 2008) }\end{array}$ & $\mathrm{h}-1,3=\frac{\mathrm{DAP}^{2}}{\beta_{0}+\beta_{1} \mathrm{DAP}+\beta_{2} \mathrm{DAP}^{2}+\beta_{3} \mathrm{DAP} \mathrm{I}}+\varepsilon_{\mathrm{i}}$ \\
Nogueira (2003) & $\ln (\mathrm{h})=\beta_{0}+\beta_{1} \mathrm{I}+\beta_{2} \mathrm{~h}_{\mathrm{dom}}+\beta_{3}\left(\mathrm{DAP}^{-1}\right)+\varepsilon_{\mathrm{i}}$ \\
Campos et al. (1984) & $\ln (\mathrm{h})=\beta_{0}+\beta_{1} \mathrm{DAP}^{-1}+\beta_{2} \operatorname{Ln}\left(\mathrm{h}_{\mathrm{dom}}\right)+\varepsilon_{\mathrm{i}}$
\end{tabular}

Em que: $\mathrm{h}$ = altura total (m); DAP = diâmetro a 1,30 metros do solo (cm); I = idade do povoamento (anos); $\mathrm{h}_{\text {dom }}=$ altura dominante $(m) ; \beta_{i}=$ coeficientes de regressão a serem estimados; $\varepsilon=$ erro.

Para avaliar a performance dos modelos ajustados foram utilizadas as seguintes estatísticas: coeficiente de determinação $\left(R^{2}\right)$, equação 5 , utilizando a soma de quadrado dos resíduos (SQres), equação 6, e a soma de quadrados totais (SQtot), equação 7. Também foram calculados o coeficiente de determinação ajustado ( $\left.R^{2} a j\right)$, equação 8 , erro padrão da estimativa (Syx), equação 9, erro padrão da estimativa em porcentagem (Syx\%) para a variável de interesse, equação 10, e análise gráfica dos resíduos (Res\%), equação 11. Para os modelos cuja variável dependente não era a variável de interesse, o erro padrão de estimativa e o coeficiente de determinação foram recalculados.

No ajuste de modelos de regressão, os coeficientes foram avaliados ao nível de $5 \%$ de probabilidade de erro pelo teste $t(p \leq 0,05)$. Caso algum coeficiente não fosse significativo, este foi excluído, e assim, o modelo foi ajustado novamente sem a variável ligada ao coeficiente. As estatísticas de ajuste e precisão para a avaliação dos modelos são apresentadas a seguir:

$$
\begin{aligned}
& \mathrm{R}^{2}=1-\frac{\text { SQres }}{\text { SQtot }} \\
& \text { SQres }=\sum_{i=1}^{n}\left(y_{i}-\hat{y}_{i}\right)^{2} \\
& \text { SQtot }=\sum_{i=1}^{n}\left(y_{i}-\bar{y}_{i}\right)^{2} \\
& R^{2} a j=1-\left(1-R^{2}\right) \cdot\left(\frac{n-1}{n-p}\right) \\
& S_{y x}=\sqrt{\frac{S Q r e s}{n-p}} \\
& S_{y x}(\%)=\left(\frac{S_{y x}}{\bar{Y}}\right) \cdot 100 \\
& \text { Res } \%=\left(\frac{y_{i}-\hat{y}_{i}}{y_{i}}\right) \cdot 100
\end{aligned}
$$


Em que: $y_{i}=$ Variável dependente observada na i-ésima árvore; $\hat{y}_{\mathrm{i}}=$ Estimativa da variável dependente na i-ésima árvore; $\bar{y}_{i}=$ Valor médio dos valores observados na i-ésima árvore; $\mathrm{p}=$ Número de coeficientes do modelo; $\mathrm{n}=$ Número de observações; SQres = Soma de quadrados de resíduos; SQtot = Soma de quadrados total.

\section{Teste de Identidade}

Após o ajuste dos modelos hipsométricos, foi selecionado o modelo mais adequado conforme as estatísticas de avaliação, para o qual foi empregado o teste de identidade de Graybill, conforme proposto por Regazzi (1996). O teste foi aplicado a fim de determinar se uma única equação poderia ser utilizada para representar o conjunto de dados dos diferentes grupos de genótipos dentro e entre os locais avaliados. O teste de identidade é baseado no teste $F$, que quando significativo ao nível de $5 \%$ probabilidade de erro, deve-se optar pelo modelo completo (rejeita-se H0) mas, caso contrário (teste F não-significativo), deve-se optar pelo modelo reduzido (aceita-se $\mathrm{HO}$ ).

\section{RESULTADOS E DISCUSSÃO}

Os coeficientes estimados para os quatro modelos testados e suas respectivas estatísticas dos grupos de genótipos 1, 2 e 3 do Local 1 estão na Tabela 3. O coeficiente de determinação ajustado $\left(R^{2}{ }_{a j}\right)$ variou de 0,96 a 0,98 , e o erro padrão de estimativa em porcentagem $\left(\mathrm{S}_{\mathrm{yx}} \%\right)$ de 5,4 a 11,2\%. Para os diferentes genótipos do Local 1, diferentes modelos tiveram melhor ajuste de acordo com as estatísticas de avaliação. Todos os coeficientes foram significativos pelo teste $t(p \leq 0,05)$.

Tabela 3. Modelos hipsométricos ajustados para os grupos de genótipos de Eucalyptus grandis, Eucalyptus grandis x Eucalyptus urophylla e Eucalyptus urophylla x Eucalyptus grandis no Local 1.

\begin{tabular}{|c|c|c|c|c|c|c|c|}
\hline \multicolumn{8}{|c|}{ Local 1 - grupo 1} \\
\hline Modelos & $\beta_{0}$ & $\beta_{1}$ & $\beta_{2}$ & $\beta_{3}$ & $\mathbf{R}^{2}{ }_{\mathrm{aj}}$ & $S_{y x}(m)$ & $S_{y x} \%$ \\
\hline Curtis (1967) & 4,0266 & $-11,5661$ & $-19,0378$ & 92,6073 & 0,96 & 1,32 & 8,5 \\
\hline Nogueira (2003) & 2,2118 & $-0,0062$ & 0,0602 & $-4,1074$ & 0,98 & 1,04 & 6,7 \\
\hline Prodan modificado (Santana, 2008) & 4,1007 & 0,5329 & 0,0242 & $-0,0062$ & 0,97 & 1,28 & 8,2 \\
\hline Campos et al. (1984) & 0,7359 & $-2,5327$ & 0,7916 & - & 0,98 & 1,05 & 6,7 \\
\hline \multicolumn{8}{|c|}{ Local 1 - grupo 2} \\
\hline Modelos & $\beta_{0}$ & $\beta_{1}$ & $\beta_{2}$ & $\beta_{3}$ & $\mathbf{R}^{2}$ aj & $S_{y x}(m)$ & $S_{y x} \%$ \\
\hline Curtis (1967) & 4,0128 & $-9,0078$ & $-22,4718$ & 80,7257 & 0,97 & 1,33 & 7,1 \\
\hline Nogueira (2003) & 2,0939 & $-0,0094$ & 0,0696 & $-3,2448$ & 0,98 & 2,07 & 11,2 \\
\hline Prodan modificado (Santana, 2008) & 3,9685 & 0,4266 & 0,0338 & $-0,0081$ & 0,97 & 1,33 & 7,2 \\
\hline Campos et al. (1984) & 0,3920 & $-1,6642$ & 0,8954 & - & 0,98 & 1,01 & 5,4 \\
\hline \multicolumn{8}{|c|}{ Local 1 - grupo 3} \\
\hline Modelos & $\beta_{0}$ & $\beta_{1}$ & $\beta_{2}$ & $\beta_{3}$ & $\mathbf{R}_{\text {aj }}^{2}$ & $S_{y x}(m)$ & $S_{y x} \%$ \\
\hline Curtis (1967) & 3,9904 & $-11,5780$ & $-18,3115$ & 89,6441 & 0,96 & 1,39 & 9,0 \\
\hline Nogueira (2003) & 2,4180 & 0,0002 & 0,0402 & $-5,0244$ & 0,97 & 1,23 & 7,9 \\
\hline Prodan modificado (Santana, 2008) & 4,8053 & 0,4053 & 0,0356 & $-0,0085$ & 0,97 & 1,31 & 8,5 \\
\hline Campos et al. (1984) & 0,9582 & $-3,0392$ & 0,7298 & - & 0,96 & 1,38 & 8,9 \\
\hline
\end{tabular}

$\beta_{i}=$ coeficientes dos modelos; $R^{2}$ aj = coeficiente de determinação ajustado; $S_{y x}=$ erro padrão d estimativa absoluto $(m) ;$ $\mathrm{S} y \times \%$ e erro padrão de estimativa relativo.

Os ajustes apresentaram elevado coeficiente de determinação ajustado para todos os grupos de genótipos, o que indica que todos os modelos explicam adequadamente a relação $\mathrm{h}$ versus DAP, I e $\mathrm{h}_{\text {dom. }}$. Os resultados das estatísticas de avaliação dos modelos ajustados para os diferentes grupos nas regiões avaliadas neste estudo são, consideravelmente, superiores aos resultados observados em plantios de Eucalyptus grandis na região dos Campos Gerais, Paraná, onde Retslaff etal. (2015) observaram coeficientes de determinação de 0,91; 
0,92 e 0,95 para os modelos de Prodan modificado (2008), Curtis (1967) e Nogueira (2003), respectivamente. No mesmo estudo, os autores verificaram erros padrão de estimativa variando de 9,4 a 11,5\%. Na região centro-oeste do Brasil, Jesus et al. (2015), após testarem modelos hipsométricos para um híbrido clonal de Eucalyptus urophylla x Eucalyptus grandis, obtiveram um coeficiente de determinação máximo de 0,75 . Os bons ajustes observados para os plantios clonais nos dois Departamentos do Paraguai são explicados, principalmente, pelo número de pares de observações de $\mathrm{h}$ e DAP ao longo das avaliações. A influência do número de árvores amostradas na precisão de estimativas de altura é destacada por Oliveira et al. (2016), os quais indicaram ainda que o número de árvores amostradas pode interferir no uso de um ou outro modelo para cada situação.

Considerando o coeficiente de determinação ajustado e o erro padrão de estimativa em porcentagem no Local 1, as melhores estatísticas de ajuste para o Grupo 1 foram dos modelos de Nogueira (2003) e de Campos et al. (1984), já para o Grupo 2 o modelo Campos et al. (1984) foi adequado pelo baixo erro padrão. Por fim, a relação hipsométrica no grupo 3 foi melhor explicada pelo modelo de Nogueira (2003).

Na Figura 3 são apresentados os gráficos de distribuição dos resíduos para os quatros modelos testados nos 3 grupos. A distribuição dos resíduos do modelo de Campos et al. (1984) não apresentou tendenciosidades nos três grupos de genótipos do local 1, com os resíduos distribuídos homegeneamente em torno da linha de regresssão. Porém, é possível observar a presença de indivíduos com altura superestimada pelos modelos com a altura dominante como variável independente. Isso é devido a presença de árvores com menor desenvolvimento, ou seja, com altura abaixo da altura padrão dos plantios. Dessa forma,

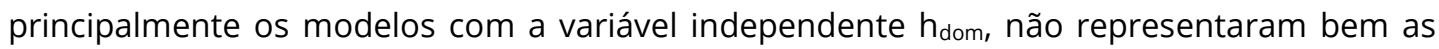
árvores com menores desenvolvimento.

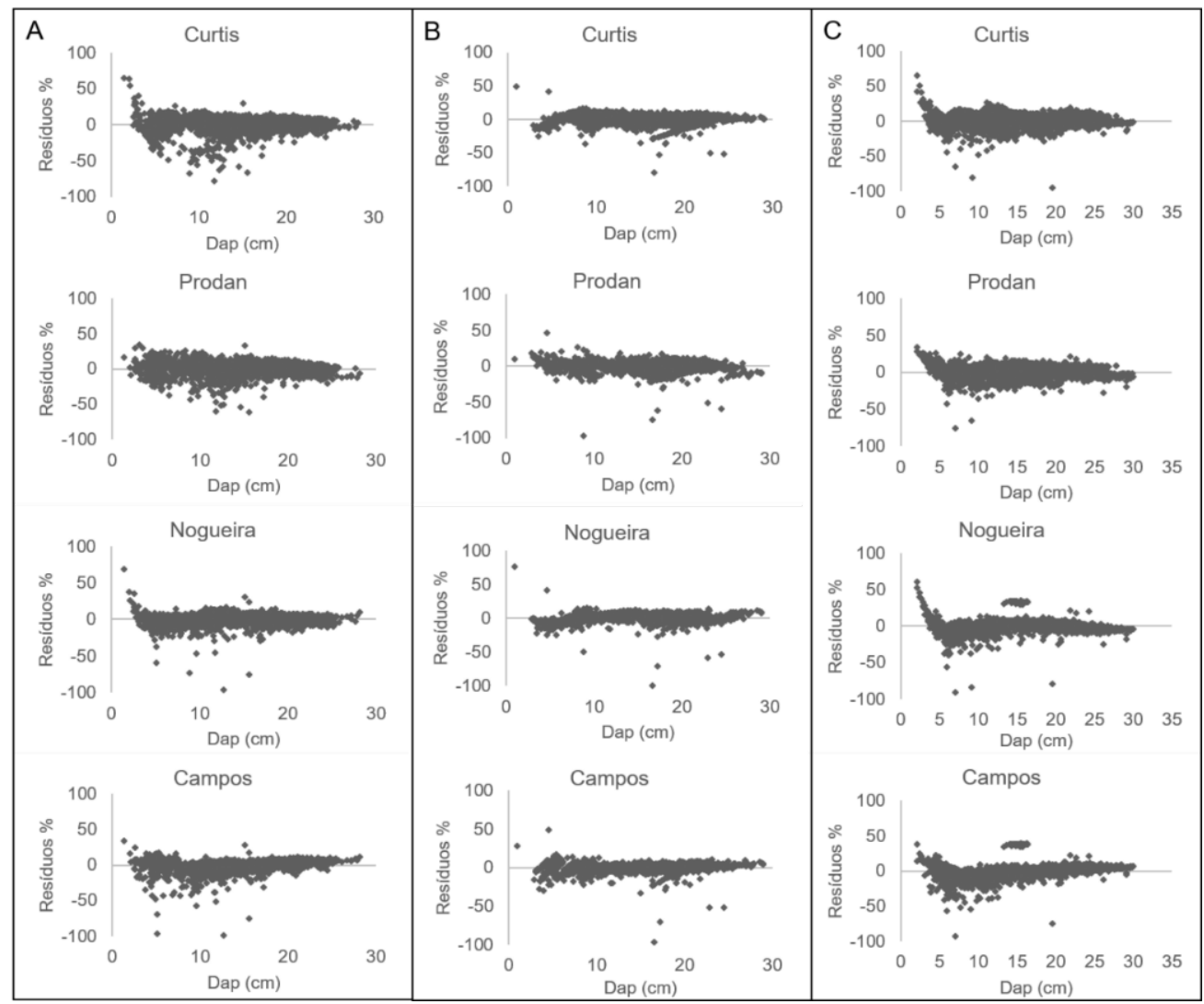

Figura 3. Distribuição dos resíduos em função do diâmetro à altura do peito (DAP em cm) para (A) Grupo de clones de Eucalyptus grandis, (B) Grupo de clones de Eucalyptus grandis x Eucalyptus urophylla e (C) Grupo de clones de Eucalyptus urophylla x Eucalyptus grandis no Local 1. 
Na Tabela 4 são apresentados os coeficientes e as estatisticas de ajuste dos modelos aplicados aos genótipos no Local 2. Para os três grupos de genótipos o modelo de Campos et al. (1984) teve as estatísticas mais acuradas, com coeficientes de determinação ajustado $\left(R^{2}{ }_{\text {aj }}\right.$ ) entre 0,98 e 0,99 e erro padrão de estimativa em porcetagem menor que $7,2 \%$. Todos os coeficientes foram significativos pelo teste $t(p \leq 0,05)$.

Tabela 4. Modelos hipsométricos ajustados para os grupos de genótipos de Eucalyptus grandis, Eucalyptus grandis x Eucalyptus urophylla e Eucalyptus urophylla x Eucalyptus grandis no Local 2.

\begin{tabular}{|c|c|c|c|c|c|c|c|}
\hline \multicolumn{8}{|c|}{ Local 2 - grupo 1} \\
\hline Modelos & $\beta_{0}$ & $\beta_{1}$ & $\beta_{2}$ & $\beta_{3}$ & $\mathbf{R}^{2}{ }_{\text {aj }}$ & $S_{y x}(m)$ & $S_{y x} \%$ \\
\hline Curtis (1967) & 4,0008 & $-10,4008$ & $-20,4700$ & 85,0289 & 0,95 & 1,70 & 10,1 \\
\hline Nogueira (2003) & 2,2657 & $-0,0104$ & 0,0668 & $-4,7268$ & 0,97 & 1,47 & 8,7 \\
\hline Prodan modificado (Santana, 2008) & 5,3990 & 0,4100 & 0,0297 & $-0,0068$ & 0,96 & 1,63 & 9,6 \\
\hline Campos et al. (1984) & 0,7418 & $-3,0242$ & 0,8018 & - & 0,98 & 1,21 & 7,2 \\
\hline \multicolumn{8}{|c|}{ Local 2 - grupo 2} \\
\hline Modelos & $\beta_{0}$ & $\beta_{1}$ & $\boldsymbol{\beta}_{2}$ & $\beta_{3}$ & $\mathbf{R}_{\text {aj }}$ & $S_{y x}(m)$ & $S_{y x} \%$ \\
\hline Curtis (1967) & 4,1050 & $-11,7493$ & $-20,7489$ & 98,7853 & 0,97 & 1,38 & 7,4 \\
\hline Nogueira (2003) & 2,3696 & $-0,0061$ & 0,0543 & $-4,7310$ & 0,98 & 1,12 & 6,0 \\
\hline Prodan modificado (Santana, 2008) & 4,1501 & 0,4709 & 0,0269 & $-0,0065$ & 0,97 & 1,41 & 7,6 \\
\hline Campos et al. (1984) & 0,6058 & $-2,3174$ & 0,8398 & - & 0,99 & 1,00 & 5,4 \\
\hline \multicolumn{8}{|c|}{ Local 2 - grupo 3} \\
\hline Modelos & $\beta_{0}$ & $\beta_{1}$ & $\beta_{2}$ & $\beta_{3}$ & $\mathbf{R}^{2}{ }_{\mathrm{aj}}$ & $S_{y x}(m)$ & $S_{\mathrm{yx}} \%$ \\
\hline Curtis (1967) & 3,9668 & $-11,0142$ & $-19,6264$ & 94,6579 & 0,95 & 1,67 & 9,7 \\
\hline Nogueira (2003) & 2,3188 & $-0,0055$ & 0,0554 & $-4,6362$ & 0,98 & 1,07 & 6,2 \\
\hline Prodan modificado (Santana, 2008) & 4,8154 & 0,3481 & 0,0432 & $-0,0098$ & 0,96 & 1,56 & 9,1 \\
\hline Campos et al. (1984) & 0,6473 & $-2,5595$ & 0,8254 & - & 0,98 & 1,01 & 5,9 \\
\hline
\end{tabular}

$\beta_{\mathrm{i}}=$ coeficientes dos modelos; $R^{2}=$ coeficiente de determinação; $R^{2}{ }_{a j}=$ coeficiente de $f$ ajustado; $S_{y x}=$ erro padrão de estimativa absoluto $(\mathrm{m}) ; \mathrm{S}$ yx\% $\%$ erro padrão da estimativa relativo.

As estatísticas de ajuste para os grupos de genótipos tanto no Local 1 quanto no Local 2 foram próximas. O ajuste satisfatório dos modelos hipsométricos deve-se, possivelmente, ao fato da inclusão das variáveis $h_{\text {dom }}$ e idade e, também devido ao conjunto de dados ser proveniente de povoamentos clonais e com manejo uniforme (Leite \& Andrade, 2003; Ribeiro et al., 2010; Oliveira et al., 2015). Na Figura 4 são apresentadas as distribuições de resíduos para os quatros modelos testados nos 3 grupos de genótipos do local 2 . Os resultados foram semelhantes ao observados para o Local 1, com o modelo de Campos et al. (1984) não apresentando tendenciosidades nos três grupos de genótipos do Local 2. Porém, também foi observado que para árvores com menor desenvolvimento em DAP e h, o modelo de Campos et al. (1984) não foi capaz de representar a altura destas árvores. Isso pode ocorrer pois, em alguns casos, a relação $\mathrm{h} / \mathrm{d}$ pode não ser representada corretamente pelos modelos, quando estes são aplicados ao conjunto de parcelas amostradas (Leite \& Andrade, 2003). 


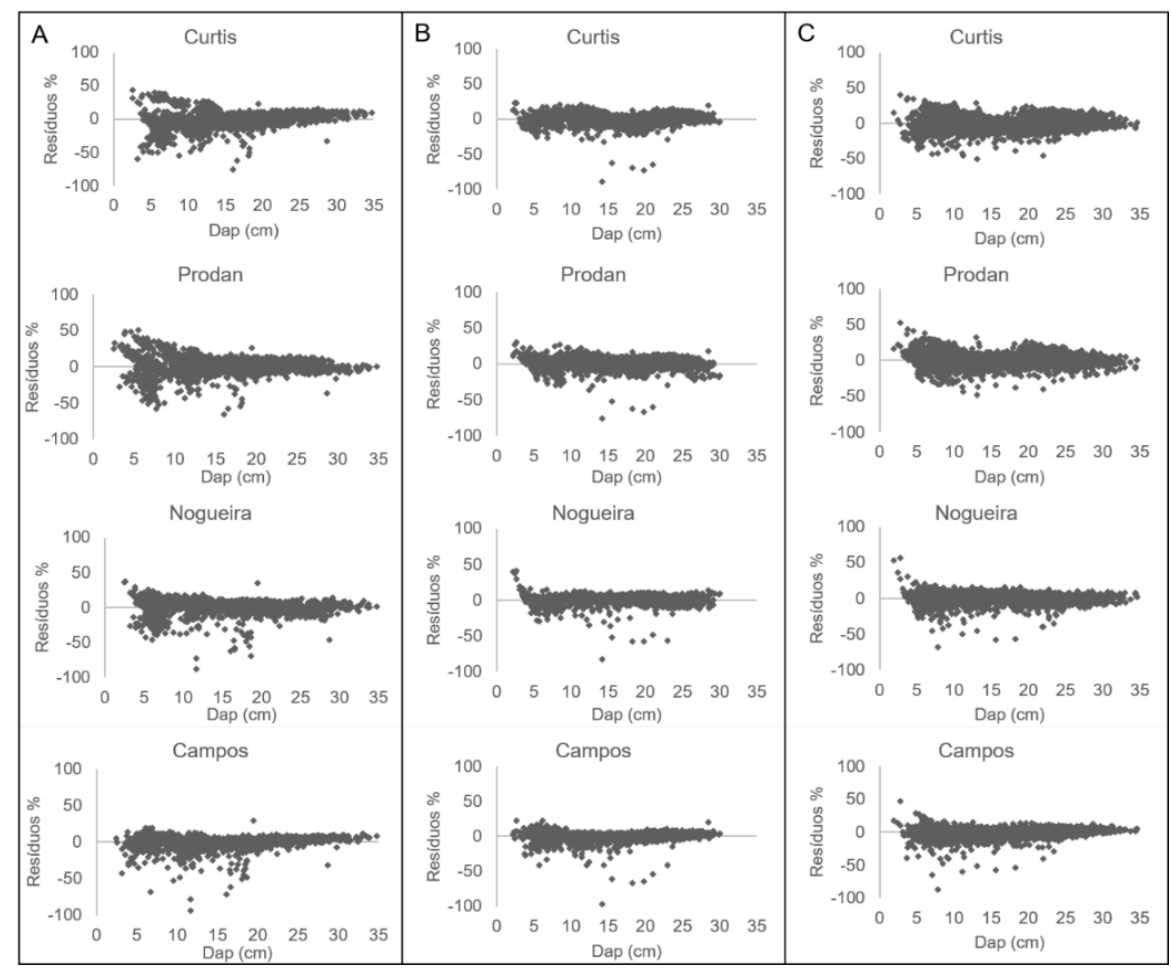

Figura 4. Distribuição dos resíduos em função do diâmetro à altura do peito (DAP em cm) para (A) Grupo de clones de Eucalyptus grandis, (B) Grupo de clones de Eucalyptus grandis x Eucalyptus urophylla e (C) Grupo de clones de Eucalyptus urophylla x Eucalyptus grandis no Local 2.

Os demais modelos apresentaram tendência de superestimar e subestimar as alturas em diâmetros menores que $20 \mathrm{~cm}$, principalmente para o Grupo 1. Superestimação de alturas em menores classes de diâmetro, pode ser atribuída a maior variabilidade de altura total em plantios jovens (Miranda et al., 2014).

Os modelos testados neste estudo estimaram adequadamente as alturas dos genótipos de eucalipto, mesmo em espaço maior ao usual no cultivo de Eucalyptus spp. em outras regiões. Em comparação, após ajuste de diversos modelos hipsométricos para Eucalyptus cloeziana e híbrido Eucalyptus urophylla $\times$ Eucalyptus grandis em sistema silvipastoril com espaçamento entre árvores de $2 \mathrm{~m} \times 2 \mathrm{~m}$ e entre renques de $12 \mathrm{~m}$ e $22 \mathrm{~m}$, Moraes Neto et al. (2010) obtiveram ajustes com coeficiente de determinação máximo de 0,98 para Eucalyptus cloeziana e de 0,95 para Eucalyptus urophylla x Eucalyptus grandis, mas com erro padrão da estimativa acima de $11 \%$.

Tanto no Local 1 como no Local 2 os modelos de Curtis (1967) e Prodan modificado (2008) mostraram resíduos com tendência de superestimar e subestimar as alturas nos menores diâmetros. Em contraste, para um híbrido de Eucalyptus urophylla $\times$ Eucalyptus grandis, os modelos de Prodan e de Curtis (1967) foram indicados pela precisão dos ajustes na região Centro-Oeste do Brasil, com resíduos sem tendências, oscilando entre $\pm 20 \%$, (Jesus et al. 2015). No estudo de Sousa et al. (2013) o modelo de Prodan também foi indicado para plantios de E. urophylla conduzido sob manejo de talhadia. Por outro lado, para clones de eucaliptos Miranda et al. (2014) também observaram imprecisão e tendências para o modelo de Campos et al. (1984) em superestimar algumas alturas. No presente estudo, observou-se que o modelo de Curtis (1967) apresentou para os Grupos 1 e 3 maior amplitude de resíduos nos diâmetros menores a $15 \mathrm{~cm}$ nos dois locais. O modelo de Curtis (1967) no estudo de Silva et al. (2015), também não apresentou ajustes adequados para estimar a altura de clones de eucalipto de 6 a 7 anos de idade.

Apesar de apresentarem estatísticas de ajuste consideradas adequadas, observou-se desvantagem para os modelos de Curtis (1967) e Prodan modificado (2008) nos resultados de $\mathrm{R}^{2}{ }_{\text {aj }}$ e $\mathrm{Syx} \%$ em relação ao modelo de Nogueira (2003), e principalmente, ao modelo de 
Campos et al. (1984), sendo que este último é indicado por Campos \& Leite (2013) para dados de inventário florestal contínuo.

Além disso, Campos \& Leite (2013) salientam que os modelos com mais variáveis independentes, entre elas a idade, índice de sítio ou altura dominante, resultam em uma melhor precisão para estimar a altura total. Sobre a inclusão de variáveis nos modelos, Leite \& Andrade (2003) questionavam a efetividade do uso da variável $h_{\text {dom }}$ para estimar a altura, mas observaram que esta variável gera estimativas mais precisas. Neste estudo, foi possível observar que os modelos de Campos et al. (1984) e Nogueira (2003), utilizando as variáveis DAP, I e $h_{\text {dom, }}$ tiveram as melhores estatísticas de ajuste e dispersão de dados.

Entre os grupos de genótipos e locais, o modelo de Nogueira (2003) apresentou o melhor ajuste apenas para grupo 1 no Local 1, com as mesmas estatísticas do modelo de Campos et al. (1984). Avaliando os modelos de Campos et al. (1984) e Nogueira (2003) para dados de inventário contínuo de um clone de E. grandis x E. urophylla, Miranda et al. (2014) observaram melhor performance do modelo de Campos et al. (1984) em relação ao modelo de Nogueira, com R ${ }_{\text {aj }}$ de 0,94 e 0,93 e Syx de 5,31 e 5,61, respectivamente. No entanto, no mesmo estudo, os autores observaram que o modelo com mais variáveis independentes teve melhores estatísticas para estimar as alturas que os modelos de Campos et al. (1984) e Nogueira (2003).

Assim, com base nas estatísticas de ajuste e na dispersão gráfica de resíduos, o modelo de Campos et al. (1984) foi selecionado para o teste de identidade. Após a aplicação do teste de identidade, concluiu-se não haver possibilidade do uso de uma equação reduzida, baseado no ajuste do modelo de Campos et al. (1984), para os grupos de genótipos, tanto no Local 1 quanto no Local 2. Além disso, foi avaliada a possibilidade de uma equação comum entre os mesmos genótipos dos dois locais avaliados. No entanto, o teste $\mathrm{F}$ significativo também indicou não ser possível o uso de uma única equação nessas condições (Tabela 5).

Tabela 5. Teste de identidade dos modelos ajustados entre grupos dos mesmos locais e entre os locais.

\begin{tabular}{|c|c|c|c|c|c|}
\hline \multicolumn{6}{|c|}{ Local 1} \\
\hline Modelo de Campos et al. (1984) & $\mathbf{R}^{2} \mathbf{a j}$ & $S_{y x}\left(m^{3}\right)$ & $S_{y x}(\%)$ & F calculado & p-valor \\
\hline G1 & 0,977 & 1,05 & 6,7 & - & - \\
\hline G2 & 0,982 & 1,01 & 5,4 & - & - \\
\hline G3 & 0,965 & 1,38 & 8,9 & - & - \\
\hline G1 e G2 & 0,979 & 1,05 & 6,2 & 58,51 & $5,37 E-37$ \\
\hline G1 e G3 & 0,970 & 1,22 & 7,9 & 15,83 & $3,08 \mathrm{E}-10$ \\
\hline G2 e G3 & 0,973 & 1,26 & 7,5 & 63,87 & $2,90 \mathrm{E}-40$ \\
\hline G1, G2 e G3 & 0,973 & 1,262 & 7,5 & 44,71 & $8,38 \mathrm{E}-54$ \\
\hline \multicolumn{6}{|c|}{ Local 2} \\
\hline Modelo de Campos et al. (1984) & $\mathbf{R}^{2}{ }_{\mathrm{aj}}$ & $S_{y x}\left(m^{3}\right)$ & $S_{y x}(\%)$ & F calculado & p-valor \\
\hline G1 & 0,976 & 1,211 & 7,2 & - & - \\
\hline G2 & 0,986 & 1,001 & 5,4 & - & - \\
\hline G3 & 0,983 & 1,015 & 5,9 & - & - \\
\hline G1 e G2 & 0,981 & 1,127 & 6,3 & 76,68 & $2,97 \mathrm{E}-48$ \\
\hline G1 e G3 & 0,980 & 1,099 & 6,4 & 15,97 & $2,5 \mathrm{E}-10$ \\
\hline G2 e G3 & 0,984 & 1,016 & 5,7 & 36,71 & $1,82 \mathrm{E}-23$ \\
\hline G1, G2 e G3 & 0,982 & 1,081 & 6,2 & 41,90 & $1,66 \mathrm{E}-50$ \\
\hline \multicolumn{6}{|c|}{ Entre os locais } \\
\hline Modelo de Campos et al. (1984) & $\mathbf{R}^{2}{ }_{\mathrm{aj}}$ & $S_{y x}\left(m^{3}\right)$ & $S_{y x}(\%)$ & F calculado & p-valor \\
\hline L1-G1 e L2-G1 & 0,98 & 1,13 & 7,0 & 54,01 & $2,93 E-34$ \\
\hline L1-G2 e L2-G2 & 0,98 & 1,00 & 5,4 & 21,63 & $6,9 \mathrm{E}-14$ \\
\hline L1-G3 e L2-G3 & 0,97 & 1,21 & 7,3 & 58,56 & $3,16 E-37$ \\
\hline
\end{tabular}

$\mathrm{L}=\mathrm{Local} ; \mathrm{G}=$ grupo; $\beta_{\mathrm{i}}=$ coeficientes dos modelos; $\mathrm{R}^{2}=$ coeficiente de determinação; $\mathrm{R}^{2}{ }_{\mathrm{aj}}=$ coeficiente de determinação ajustado; $S_{y x}=$ erro padrão de estimativa absoluto $(m) ; S_{y x} \%=$ erro padrão da estimativa relativo. Significativo ao nível de $5 \%$ de significância. 
O modelo de Campos et al. (1984) selecionado para o teste de identidade, apresentou para todos os genótipos, nos dois locais avaliados, correlação entre as alturas observadas e estimadas maior que 0,98 , o que em alguns casos pode levar a resultados contraditórios do teste $\mathrm{F}$ (Leite \& Andrade, 2003). Porém, em função das estatísticas de ajuste e dos gráficos de resíduos, foi possível observar que o modelo de Campos et al. (1984) teve o melhor ajuste, mesmo que seja necessário o uso de diferentes equações para cada grupo de genótipo em cada local.

\section{CONCLUSÃO}

Os modelos hipsométricos testados apresentaram excelentes estatísticas de ajuste e precisão, com coeficientes de determinação superiores a 0,96 e erros médios inferiores a 11\%, sendo que o modelo de Campos et al. (1984) apresentou as melhores estatísticas. Estes resultados são decorrentes da inclusão das variáveis idade e altura dominante, além do DAP, mas também em consequência da homogeneidade dos povoamentos avaliados. Finalmente, o teste de identidade mostrou que não foi possível o uso de uma equação hipsométrica reduzida, tanto entre genótipos em cada local quanto entre os genótipos dos locais avaliados.

\section{REFERÊNCIAS BIBLIOGRÁFICAS}

Bonete, I. P., Deus, K. H. P., Benin, C. C., Garrett, A. T. A., Dias, A. N., \& Watzlawick, L. F. (2016). Identity of hypsometric and volumetric models for Eucalyptus benthamii grouped by planting spacings. Australian Journal of Basic and Applied Sciences, 10(13), 101-107.

Britos, J., \& Leguizamón, A. (2013). Manual de plantaciones Forestales. Técnicas de instalácion y manejo (18 p.). San Lorenzo: Instituto Florestal Nacional.

Campos, J. C. C., Ribeiro, J., \& Paula Neto, F. (1984). Inventário florestal nacional: reflorestamento: Minas Gerais. (126 p.). Brasília: IBDF.

Campos, J. C. C., \& Leite, H. G. (2009). Mensuração florestal. Perguntas e respostas (3. ed., 548 p.). Viçosa: Editora UFV.

Campos, J. C. C., \& Leite, H. G. (2013). Mensuração florestal. Perguntas e respostas (4. ed., 605 p.). Viçosa: Editora UFV.

Curtis, R. O. (1967). Height diameter and height diameter age equations for second growth douglas-fir. Forest Science, 13(4), 365-375.

Finger, C. A. (1992). Fundamentos de biometria florestal (269 p.). Santa Maria: UFSM/CEPEF/FATEC.

Food and Agriculture Organization of the United Nations - FAO. (2002). Estado de la informacion forestal en Paraguay. Monografía de Países (Vol. 14, 199 p.). Santiago: FAO.

Food and Agriculture Organization of the United Nations - FAO. (2018). FAOSTAT. Land use. Recuperado em 29 de setembro de 2018, de http://www.fao.org/faostat/en/\#data/RL

Frey, G. E. (2007). Rentabilidad de la inversión forestal en Paraguay (43 p.). Washington: Banco Mundial.

Instituto Forestal Nacional - INFONA. (2013). Proyecto "Promoción de plantaciones forestales" (Informe Final, 32 p.). San Lorenzo: INFONA.

Jesus, C. M., Miguel, E. P., Azevedo, G. B., Azevedo, G. T. O. S., \& Pereira, R. S. (2015). Modelagem hipsométrica em povoamento clonal de Eucalyptus urophylla $\times$ Eucalyptus grandis no Distrito Federal. Enciclopédia Biosfera, 11(21), 1928-1308.

Leite, H. G., \& Andrade, V. C. L. (2003). Importância das variáveis altura dominante e altura total em equações hipsométricas e volumétricas. Revista Árvore, 27(3), 301-310. http://dx.doi.org/10.1590/S0100-67622003000300005.

Machado, S. A., \& Figueiredo Filho, A. (2006). Dendrometria (2. ed., 316 p.). Guarapuava: Editora UNICENTRO.

Miranda, R. O. V., David, H. C., Ebling, Â. A., Môra, R., Fiorentin, L. D., \& Soares, I. D. (2014). Estratificação hipsométrica em classes de sítio e de altura total em plantios clonais de eucaliptos. Advances in Forestry Science, 1(4), 113-119.

Moraes Neto, S. P., Pulrolnik, K., Vilela, L., Munhoz, D. J. M., Gumarães Junior, R., \& Marchão, R. L. (2010). Modelos hipsométricos para Eucalyptus cloeziana e Eucalyptus urophylla x Eucalyptus grandis em 
sistema agrossilvipastoril (Boletim de Pesquisa e Desenvolvimento, 286, 34 p.). Planaltina: Embrapa Cerrados.

Nogueira, G. S. (2003). Modelagem do crescimento e da produção de povoamentos de Eucalyptus sp. e de Tectona grandis submetidos a desbaste (Tese de Doutorado em Ciência Florestal). Universidade Federal de Viçosa, Viçosa.

Oliveira, G. M. V., Mello, J. M., Altoé, T. F., Scalon, J. D., Scolforo, J. R. S., \& Pires, J. V. (2015). Equações hipsométricas para Eucalyptus spp. não manejado em idade avançada com técnicas de inclusão de covariantes. Cerne, 21(23), 483-492. http://dx.doi.org/10.1590/01047760201521031740.

Oliveira, R. R., Oliveira, X. M., Silva, G. C. C., \& Mayrinck, R. C. (2016). Acurácia de relações hipsométricas para diferentes estratégias de validação em Eucalyptus urograndis. Revista Verde de Agroecologia e Desenvolvimento Sustentável, 11(5), 123-127. http://dx.doi.org/10.18378/rvads.v11i5.3746.

Portillo, J. L. (2010). Manual para manejo de especies forestales de rápido crecimiento. Eucalypto e Grevillea (57 p). San Lorenzo: Centro Internacional de Investigación de las Ciencias Agropecuarias del Japón.

Regazzi, A. J. (1996). Teste para verificar a identidade de modelos de regressão. Pesquisa Agropecuária Brasileira, 31(1), 1-17.

Retslaff, F. A. S., Figueiredo Filho, A., Dias, A. N., Bernett, L. G., \& Figura, M. A. (2015). Curvas de sitio e relações hipsometricas para Eucalyptus grandis na região dos Campos Gerais, Paraná. Cerne, 21(2), 219-225. http://dx.doi.org/10.1590/01047760201521021349.

Ribeiro, A., Ferraz Filho, A. C., Mello, J. M., Ferreira, M. Z., Lisboa, P. M. M., \& Scolforo, J. R. S. (2010). Estratégias e metodologias de ajuste de modelos hipsométricos em plantios de Eucalyptus sp. Cerne, 16(1), 22-31. http://dx.doi.org/10.1590/S0104-77602010000100003.

Rodrigues, C. K., Lopes, E. S., Figueiredo Filho, A., \& Pelissari, A. L. (2017). Identidade de modelos hipsométricos e volumétricos de povoamentos de Pinus com diferentes idades submetidos ao desbaste mecanizado. Biofix Scientific Journal, 2(1), 54-59. http://dx.doi.org/10.5380/biofix.v2i1.51947.

Sanquetta, C. R., Watzlawick, L. F., Côrte, A. P. D., Fernandes, L. A. V., \& Siqueira, J. D. P. (2009). Inventários florestais: planejamento e execução (2. ed., 316 p.). Curitiba: Multi-Graphic Gráfica e Editora.

Santana, C. J. de O. (2008). Simulação do crescimento e da produção em plantações desbastadas de Eucalyptus grandis com diferentes procedimentos de obtenção dos parâmetros da distribuição Weibull (Dissertação de Mestrado em Engenharia Florestal) - Universidade Federal do Paraná, Curitiba.

Scolforo, H. F., Raimundo, M. R., Scolforo, J. R. S., Mello, J. M., Batista, A. P. B., \& Bullock, B. (2015). Hypsometric approaches to Eucalyptus experiments in Brazil. African Journal of Agricultural Research, 10(45), 4176-4184. http://dx.doi.org/10.5897/AJAR2015.10151.

Silva, A. J., Silva Filho, J. L. F., Silva, M. D. T., Roque, C. G., \& Cunha, F. F. (2015). Ajuste de modelos hipsométricos e volumétricos para três clones de Eucalyptus em Mineiros-GO. Nucleus, 12(2), 221 230. http://dx.doi.org/10.3738/1982.2278.1372.

Sousa, G. T. O., Azevedo, G. B., Barreto, P. A. B., \& Conceição Júnior, V. (2013). Relações hipsométricas para Eucalyptus urophylla conduzidos sob regime de alto fuste e talhadia no Sudoeste da Bahia. Scientia Plena, 9(4), 1-7.

Contribuição dos Autores: HABA: Conceituação, Metodologia, Escrita - Primeira Redação, Escrita - Revisão e Edição, Curadoria de Dados; ATAG: Escrita - Primeira Redação, Escrita - Revisão e Edição; LRL: Metodologia, Curadoria de Dados; AND: Metodologia, Escrita - Revisão e Edição, Supervisão, Curadoria de Dados; EVT: Escrita Revisão e Edição, Supervisão, Curadoria de Dados; AFF: Metodologia, Escrita - Revisão e Edição, Supervisão, Curadoria de Dados; FARG: Metodologia, Escrita - Revisão e Edição, Supervisão, Curadoria de Dados; OMVC: Conceituação, Metodologia. 